\title{
Obesity, Intestinal Gut Barrier and Microbiota: an Emerging Frontier on Energy Metabolism
}

\author{
Fernanda de Azevedo Marques Lopes ${ }^{1,2}$, Vicente Lopes da Silva Junior ${ }^{1,2}$, Ana Teresa Pugas Carvalho ${ }^{2}$, \\ Rodolfo Albano $^{3}$, Raquel Carvalho Castiglione ${ }^{4}$, Luiz Guilherme Kraemer-Aguiar ${ }^{2 *}$
}

${ }^{1}$ Postgraduate Program in Clinical and Experimental Pathophysiology (FISCLINEX), State University of Rio de Janeiro (UERJ), Rio de Janeiro, RJ, CEP 20551-030 - Brazil

${ }^{2}$ Department of Internal Medicine, Faculty of Medical Sciences, State University of Rio de Janeiro (UERJ), Rio de Janeiro, RJ, CEP 20551-030 - Brazil

${ }^{3}$ Department of Biochemistry, Roberto Alcântara Gomes Biological Institute, State University of Rio de Janeiro, Rio de Janeiro, RJ, CEP 20551-030 - Brazil

${ }^{4}$ Laboratory for Clinical and Experimental Research on Vascular Biology (BioVasc), Biomedical Center, State University of Rio de Janeiro, Rio de Janeiro, RJ, CEP 20551-030 - Brazil

*Correspondingauthor:LuizGuilhermeKraemer-Aguiar,RuaSãoFranciscoXavier,524-Pavilhão, ReitorHaroldoLisboadaCunha, térreo (104), CEP 20550-013, Rio de Janeiro, RJ, Brazil, Tel: 55-21-2334-0703; Fax 55-21-2334-0692; E-mail: gkraemer@ig.com.br

Citation: Kraemer-Aguiar, L.G., et al. Obesity, Intestinal Gut Barrier and Microbiota: An Emerging Frontier on Energy Metabolism. (2016) J Gastro Dis Liver Func 2(1): 59- 67.

\begin{abstract}
Background \& Aims:

An increasing interest in demonstrating differences between intestinal microbiota of obese and lean individuals has been observed during the last decade. The gut microbiota has been related to a number of metabolic abnormalities that could be associated not only to the pathophysiology of obesity, but also to several other highly prevalent diseases.

As researches had shown differences in the prevalence of different bacteria in the gut of these individuals much importance has been given to microbiota composition along intestinal tract. However, few studies have related these findings to changes in the morphological and functional features of the intestinal epithelium.

The intestinal barrier is a complex system and its integrity is essential to prevent against the entrance of harmful microorganisms or molecules to enter the bloodstream, such as LPS (bacterial lipopolysaccharide), a well-known trigger of the low grade inflammatory status. Increased intestinal permeability is one of the proposed mechanisms linking the gut microbiota with metabolic dysfunction, but there are few available data to define which one would be the cause or consequence. This review aims to show the current knowledge about the association between intestinal permeability in obesity and alterations in gut microbiota, in order to bring up a new vision of the pathophysiology of obesity and possibly promote future studies to better elucidate it.

Methods: Pub Med searches were performed using terms "microbiota", "gut permeability" and "obesity". Articles related to intestinal permeability were selected.

Results: Hyperactivation of endogenous cannabinoid system appears to augment gut permeability to LPS which, in turn, seems to enhance chronic inflammation status found in obesity state. Gut microbiota may play an important role in this process, through the regulation of the local immune system and also by regulating the amount of LPS which crosses the gut barrier.

Conclusions: Lypopolyssacharide, endocanabinoid system and gut microbiota seem to play an important role in the development of obesity and emerge as a possible target for the treatment of obesity and correlated diseases.
\end{abstract}

Keywords: Obesity; Gut permeability, Microbiota

Copyrights: (C) 2016 Kraemer-Aguiar, L.G. This is an Open access article distributed under the terms of Creative Commons Attribution 4.0 International License. 


\section{Introduction}

Although a few years ago the role of gut microbiota in human health was ignored, currently a well-known fact in medical sciences is that gut microbes play an important physiological and pathophysiological role in obesity, diabetes, metabolic syndrome, non-alcoholic liver disease, inflammatory bowel diseases and also some other pathologic conditions ${ }^{[1]}$.

In humans, the mechanisms by which gut microbiota influences gut homeostasis remains to be clarified, but it seems that the lipopolysaccharide (LPS), present in the wall of human gut resident bacteria, would be an important factor involved in the regulation of the integrity and function of intestinal epithelium and at unbalanced states related to diseases, it may result in increased gut barrier permeability and its outcomes ${ }^{[2]}$.

By considering obese and normal weight individuals as different from each other based solely on their anthropometric characteristics, we would be too simplistic. Normal weight and obese subjects differ not only in their anthropometry but also in many other aspects, such as inflammatory status and the predominance of some intestinal bacteria phyla ${ }^{[3]}$.

Since a long time ago, obesity is no longer considered a cosmetic problem but a complex disorder. It has been linked to a broad set of other disorders such as arterial hypertension, diabetes mellitus, nonalcoholic fat liver disease and cancer. Therefore, it could be responsible, at least in part, for the great amount of cardiovascular deaths in Western Civilization. Furthermore, obesity is related to rheumathological and respiratory diseases such as osteoarthrosis, lumbar spine pain and obstructive sleep apnea. Taken together, these reports show that obesity must be considered as a systemic disorder in excellence ${ }^{[4]}$.

Several studies have already demonstrated possible reasons for the pandemic proportions that obesity has reached, among which we could mention well-known factors such as sedentary life, meals with high energy density, larger food portions, fast-food, eating out-of-home and the psychological stress. In fact, all of them seem to play a crucial role on the pathophysiology of obesity. However, in the past decade some new findings showed some emerging factors related to obesity and its development. Perhaps the most important question that arises is: what are the factors that contribute to the maintenance of obesity and why is it so difficult to treat?

Obesity is a state of chronic subclinical systemic inflammation. Recent evidence has suggested that the gut of obese subjects has differences in host bacteria status with a higher proportion of Firmicutes while in normal weight ones, Bacteroidetes is a more prevalent phylum ${ }^{[3]}$. One of the greatest challenges for researchers on this field nowadays is to clarify whether these differences are the cause or the consequence of obesity, as well as the understanding of the mechanisms involved in this process. The role of the gut in the pathophysiological mechanisms related to obesity is a promising new research avenue that may clarify a number of these issues, not only relating to obesity, but also to other conditions, such as inflammatory bowel disease, type 2 diabetes mellitus and immune-related diseases. This brief review attempts to demonstrate on the basis of the literature data the role of gut permeability in the pathophysiology of obesity-related diseases, focusing especially on its clinical use in future treatments.

\section{Physiology of Gastrointestinal Tract}

Anatomically, the gut may be divided in two regions: the small and the large intestines. Physiologically, both parts take part in the digestion and absorption of dietary nutrients. At the duodenum, the first part of the small intestine is the place where major chemical reactions occur between partially digested food that has already suffered the action of gastric juice and bile-pancreatic secretions. From the contact between the bolus and bile secretions, fat emulsification occurs, leading to micelle formation that potentiates pancreatic lipase activity. In addition to the digestive process, a parallel effect played by GLP-1 and GIP secreting endocrine intestinal cells also occurs with important implications on satiety and energy metabolism. Finally, once the bolus reaches the large intestine, named colon, the digestion and nutrient absorption is almost completed leaving this part of the gut responsible for water absorption.

In addition to its role in digestion it is currently known that the gastrointestinal tract is a "microbiotic system" since it contains about 1010 to $1012 \mathrm{CFU} / \mathrm{ml}$ of bacteria ${ }^{[5]}$. Recent studies have demonstrated that the intestinal flora plays an important role in nutrition and also on disease, and nowadays, the term "microbiome" is used as a link between the bacterial genes and our own genome and their interplay that could interfere with metabolic processes.

\section{Obesity and gut epithelium - what is already known?}

The link between obesity and the gut reminds of clinical studies performed at the initial stages of obesity research in which scientists believed that this disease could be merely linked to the length of the small intestine suggesting a positive relationship between body adiposity and intestinal length. This theory was tested in these clinical studies that evaluated intestines from autopsies of obese and normal weight individuals revealing that the data were highly variable, depending on the method and measurements employed ${ }^{[6-9]}$.

On the $70 \mathrm{~s}$, the most important study was designed and performed by Guzman and co-workers ${ }^{[10]}$ who measured the length of the small intestine during intestinal bypass surgery in 272 morbidly obese patients during jejunoileal surgery and compared it to the length of the small intestine measured on 121 non-obese patients who had undergone partial ileal resection for treating hyperlipidemia. They provided evidence that there was no significant difference in the length of intestines between obese and non-obese patients.

In the light of these data, researchers concluded that the differences between obese and normal weight subjects were not related to the length of the small intestine itself. Therefore, another hypothesis aroused. Would the specific morphological and functional features that alter the structure of the epithelium play a role on obesity? The first experiments to investigate this were conducted on animal models and enabled us to partially prove this thesis.

In 2005, Ley and co-workers demonstrated that obese mice had some differences in the composition of their intestinal microbiota as they observed that Bacteroidetes was the least prevalent phylum in the intestines (around 50\% less) of obese animals while a higher prevalence of Firmicutes was concurrently noticed in the normal weight ones. Additionally, the investigators subjected obese animals to a weight-loss diet and showed that their microbiota became very similar to that of nor- 
mal weight mice ${ }^{[1]}$.

A similar characteristic of the intestinal microbiota was observed in humans when Ley and co-workers, in 2006, indicated that the development of obesity has a microbial component. In this study, they found that the relative proportion of Bacteroidetes is decreased in obese people when compared to lean ones and, more importantly, that this proportion increases with weight loss with different types of low-calorie diet ${ }^{[3]}$.

In one study, Backhed and collaborators compared mice with and without host bacteria in the gut, the latter group also known as germ-free animals. Mice colonized with host bacteria had increased body fat content in relation to their germ-free counterparts. Surprisingly, the animals with host bacteria had 30\% less caloric intake and a higher resting metabolism than germ-free mice. In this experimental model, these findings suggested a major role of microbiota on energy metabolism ${ }^{[12]}$. Subsequently, another group grounded this hypothesis and demonstrated by the colonization of "germ-free" mice with microbiota from obese mice a significant increase in fat mass compared to those mice colonized with microbiota from lean ones ${ }^{[13]}$. However, as shown in table 1 some contradictions still exist. A possible explanation for this could be related to the differences in the methods employed to detect these bacteria. Moreover, although the majority of the results have differences in the proportion of bacterial groups, it is still not possible to identify whether any group of specific microorganisms directly contributes to the development of obesity and metabolic disorders $^{[14]}$.

Table 1: Alterations detected in the human gastrointestinal microbiota in obese and overweight subjects. Results from comparisons between obese or overweight subjects with normal weight controls

\begin{tabular}{|c|c|c|c|c|c|c|c|c|c|c|c|c|c|c|c|c|c|c|}
\hline \multirow[b]{3}{*}{ Reference } & \multicolumn{18}{|c|}{ Bacterialgrouporspecies } \\
\hline & \multicolumn{6}{|c|}{ Bacteroidetes } & \multicolumn{12}{|c|}{ Firmicutes } \\
\hline & 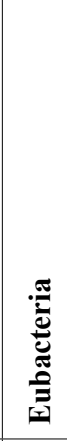 & 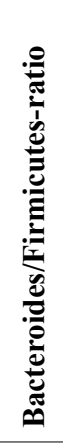 & 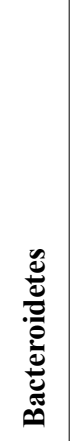 & 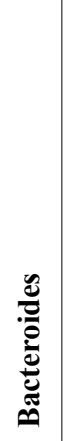 & 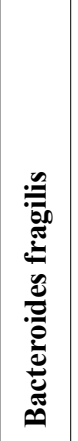 & 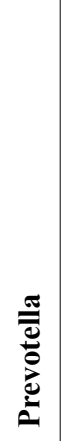 & 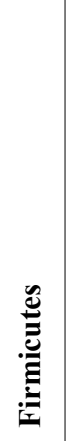 & 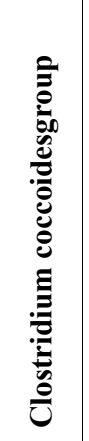 & 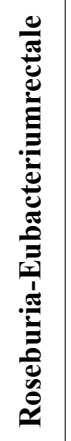 & 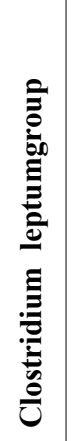 & 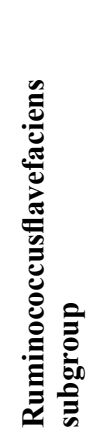 & 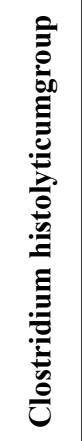 & 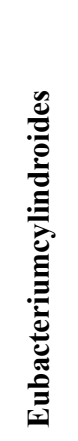 & 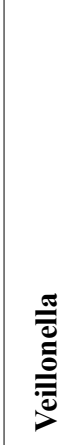 & 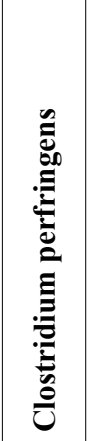 & 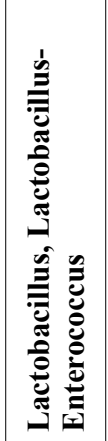 & 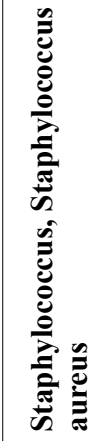 & 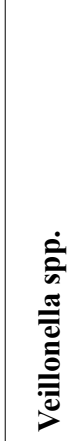 \\
\hline Armougomet al., 2009 & & & $\downarrow$ & & & & $\leftrightarrow$ & & & & & & & & & $\uparrow$ & & \\
\hline Colladoet al., 2008 & $\leftrightarrow$ & & & $\uparrow$ & $\leftrightarrow$ & & & & & & & $\leftrightarrow$ & & & & & $\uparrow$ & \\
\hline Duncan et al., 2008 & $\leftrightarrow$ & & & $\leftrightarrow$ & & & & & & & & & & & & & & \\
\hline Larsenet al., 2010 & & $\leftrightarrow$ & & & & & & & & & & & & & & & & \\
\hline Leyet al., 2006 & & $\downarrow$ & $\downarrow$ & & & & $\uparrow$ & & & & & & & & & & & \\
\hline Malinenet al., 2010 & $\leftrightarrow$ & & & & & & & $\leftrightarrow$ & & & & & & & & $\leftrightarrow$ & & $\leftrightarrow$ \\
\hline Santacruzet al., 2010 & $\leftrightarrow$ & & & $\downarrow$ & & & & $\leftrightarrow$ & & $\leftrightarrow$ & & & & & & $\uparrow$ & $\uparrow$ & \\
\hline Schwiertzet al., 2010 & $\leftrightarrow$ & $\uparrow$ & $\uparrow$ & $\uparrow$ & & $\leftrightarrow$ & $\downarrow$ & $\leftrightarrow$ & & $\downarrow$ & $\downarrow$ & & $\leftrightarrow$ & $\leftrightarrow$ & & $\leftrightarrow$ & & $\leftrightarrow$ \\
\hline Tiihonenet al., 2010 & $\leftrightarrow$ & & $\leftrightarrow$ & & & & & $\leftrightarrow$ & & & & & & & $\leftrightarrow$ & $\leftrightarrow$ & & \\
\hline Turnbaughet al., 2009 & & & $\downarrow$ & & & & $\leftrightarrow$ & & & & & & & & & & & \\
\hline Zhang et al., 2009 & & & $\leftrightarrow$ & & & $\uparrow$ & & & & & & & & & & & & \\
\hline
\end{tabular}

Studies on microbiome have expanded in order to elucidate its role in a great number of other diseases. Fecal microbiota transplantation is a successful therapy for patients with refractory Clostridium difficile infections ${ }^{[15]}$ and it has also been suggested as a treatment option for inflammatory bowel disease, given a role for intestinal microbiota in this disease ${ }^{[16,17]}$. Vermeire and colleagues assessed the impact of fecal microbiota transplantation in patients with inflammatory bowel disease and studied predictors of clinical (non)response in microbial profiles of donors and patients and concluded that fecal microbiota transplantation led to endoscopic and long-term ( $>2$ years) remission in 2 out of 8 ulcerative colitis patients ${ }^{[16]}$.

Many other experimental and clinical studies that have been published in the last years were able to confirm that the composition of the intestinal flora shows differences between lean and obese subjects ${ }^{[1-22]}$. However, there is still one not well elucidated point: how are these changes related to obesity?

Over the years, many mechanisms have been proposed in an attempt to explain this relationship. Possible mechanisms relating obesity to intestinal microbiome are depicted in figure 1 . The most relevant ones suggest that gut flora may influence many aspects of gut homeostasis such as the capacity for energy extraction from food, the regulation of host genes that modulate 
how energy could be used or stored and the modulation of LPS levels that leads to a chronic local and systemic subclinical inflammation, to a prolonged time in gut transit time and also to an altered permeability of the intestinal epithelium ${ }^{[5,23,24]}$. All of these mechanisms would converge to increase the likelihood of developing obesity among individuals who show higher gut permeability that would enable LPS to cross the gut barrier in great amounts and reach the blood stream, causing low grade systemic inflammation, a subsequent altered regulation of energy metabolism and hunger-satiety homeostasis and weight gain.

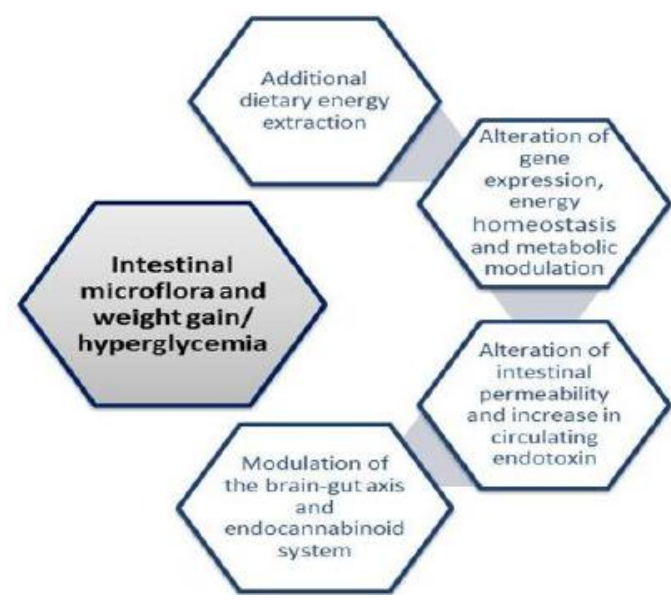

Figure 1: Proposed mechanisms linking obesity and intestinal microbiota

\section{Additional dietary energy extraction}

One hypothesis proposed to explain why greater energy extraction from food by bacteria could be involved in obesity pathophysiology is related to the transfer of hydrogens. Oxidation of this molecule increases the formation of methane which, in turn, facilitates the fermentation of polysaccharides from vegetables and other sources. The accelerated fermentation stimulates hydrolysis of organic material that normally cannot be digested, leading them free for the production of acetate, which can be absorbed and easily used by the host. It has been shown that obese subjects have greater amount of Archea and Provotellacea which indicates that this microbiota would favor the formation of methane ${ }^{[25]}$ and the above mentioned cycle.

Increments in intestinal microvasculature may result in increased intestinal nutrients absorption. It was demonstrated that, by exposing germ free animals to bacteria, and turning them into colonized animals, an increased intestinal microvasculature occurred possibly due to interactions of bacteria with the host immune system cells ${ }^{[26]}$.

Another important aspect is related to differences in the production of acetate and propionate according to host microbiota. Germ-free animals inoculated with microbiota from obese animals produced greater amounts of acetate and propionate than those inoculated with microbiota from lean animals ${ }^{[13]}$. The resultant of this inoculation was a greater weight gain on the former animals. In addition, it was shown that the consumption of diet with high proportions of fat and sugar, those classically used to induce obesity in animal models, did not cause obesity in germ-free animals ${ }^{[27]}$. As a counterpoint, the hypothesis that the absence of microbials in germ free animals would give protection against weight gain was challenged by Fleissner and coworkers $^{[28]}$. They have shown that the fatty acid profile was more important than the amount of macronutrients in determining the extent of protection to weight gain by the germ free animals, which disputes the additional dietary energy extraction hypothesis and makes this issue more complex than previously thought ${ }^{[29]}$.

\section{Alteration of gene expression, energy homeostasis and met- abolic modulation}

The ectopic fat accumulation in sites, such as the liver and the skeletal muscle, influences the balance of glycidic and lipidic metabolism, through the development of insulin resistance ${ }^{[30,31]}$. There is evidence that the microbiota plays an important role in the regulation of intermediary metabolism in the host influencing gene expression in these organs ${ }^{[32]}$.

Microbiota studies have shown some alterations in intestinal expression of FIAF (named fasting-induced adipose factor) protein that interferes with the metabolism of intestinal fatty acids ${ }^{[12]}$. Additionally, another study showed changes in gene expression of carbohydrate response element-binding protein (ChREBP) and sterol regulatory element-binding protein 1 (SREBP-1), which are both nuclear regulators of lipogenic enzymes, able to favor the triacylglycerol accumulation in the liver tissue $^{[33]}$.

\section{The Endocannabinoid System}

The endocannabinoid system (eCB) is the machinery formed by the two main endocannabinoids - anandamide and 2-arachidonoyl glycerol (2AG), their receptors (CB1 and CB2) and enzymes involved in the biosynthesis and degradation of its ligands. The components of this system are expressed from the brain to several other tissues, including liver and gastrointestinal tract. Endocannabinoids are synthesized from arachidonic acid and are rapidly hydrolyzed to inactive compounds by catalytic action of specific enzymes ${ }^{[34]}$. CB1 receptor is the best characterized target of exogenous and endogenous cannabinoids in the modulation of neuroendocrine and metabolic responses. Its expression has already been demonstrated in a variety of tissues such as adipocytes, muscle and liver ${ }^{[35-37]}$. Additionally, the eCB is present in the gastrointestinal tract, where it has an important action on modulation of motility, inflammation and secretion ${ }^{[38]}$.

Endocannabinoids are very lipophilic, so they cannot be stored in vesicles like insulin and neurotransmitters. As a result of this, the regulation of the eCB is tightly controlled by their synthesis, release, uptake and degradation ${ }^{[34]}$, suggesting that its action occurs on demand.

Any anandamide (firstly identified in 1992) such as 2AG (identified in 1995) modulates neuronal activity through the backward elimination process of neurotransmitter release. In the central nervous system, endocannabinoids can act as neurotransmitters when post-synaptic release of endocannabinoids targets pre-synaptic neurons and activates $\mathrm{CB} 1$ receptor, in a process known as retrograde signaling ${ }^{[39,40]}$. In this process, the action of neurotransmitters at post-synaptic neurons stimulates the rapid and transient production of endocannabinoids on demand from phospholipid precursors present in the cell membrane. The overall effect is a decrease in the release of neurotransmitters such as glutamate and GABA but, in fact, the final result of the action of endocannabinoids depends on whether the specific synapse is an excitatory or inhibitory one, resulting respectively in suppression or release of neuronal transmission ${ }^{[41]}$. 
The CB1 receptor is the one that is related to anabolic functions of endocannabinoids, with broad and abundant distribution not only in the brain but also in a variety of tissues. The action of endocannabinoids on CB1 receptor results in an increased appetite, weight gain, lipogenesis and higher insulin sensitivity ${ }^{[42]}$. In the hypothalamus, endocannabinoids increase the production of orexigenic neurotransmitters while reducing anorexigenic ones. In the reward center, endocannabinoids promote motivation to demand and food consumption and increase for the consumption of highly palatable foods. In the brainstem, they block the nausea and satiety signals transmitted from the vagus nerve. Peripherally, endocannabinoids facilitate the absorption of nutrients in the gut, stimulate lipogenesis and compromise the glucose uptake in muscle. It was recently shown that the liver also expressed $\mathrm{CB} 1$ receptors, promoting the induction of Steroid Regulator Element Binding Protein-1 (SREBP-1) and, consequently, hepatic lipogenesis ${ }^{[37]}$. Molecular mediators such as SREBP-1 are involved in the pathophysiology of steatosis and hepatic injury in states of obesity and insulin resistance ${ }^{[43]}$. This is a transcription factor that acts in the cascade of hepatic lipogenesis via transcription of the genes of key enzymes such as acetyl-CoA carboxylase-1 (ACC1) and fatty acid synthase $(\mathrm{FAS})^{[37,44]}$

Some studies found that obesity is characterized by greater eCB tone. This finding could be verified by eCB plasma levels, by an altered expression of the $\mathrm{CB} 1$ receptor and also by increased eCB levels in adipose tissue ${ }^{[35,45-47]}$. Interestingly, it had already been demonstrated that LPS was able to control the synthesis of eCB components both in vivo ${ }^{[48]}$ and in vitro ${ }^{[49]}$ through mechanisms that are dependent on LPS receptor signaling ${ }^{[50]}$.

One study demonstrated that obese mice treated with the CB1 receptor antagonist (SR141716A) for 12 days exhibited reduced gut permeability as shown by their reduced plasma LPS levels compared to controls. Moreover, it was found that this reduction was possible through a mechanism linked to the improvement of gut barrier function which was inferred from changes in the distribution and localization of tight junction proteins [zonula ocludens -1 (ZO1) and occludin] ${ }^{[51]}$. In the same study, the authors also found that the reduction on gut permeability was associated with decreased expression of the hepatic inflammatory markers tumour necrosis factor- $\alpha$, plasminogen-activator inhibitor- 1 and toll-like receptor- 4 mRNA. Other studies have also shown that in vivo colonic CB1 mRNA expression was reduced in gut microbiota models known to improve gut permeability and that mice treated with a $\mathrm{CB} 1$ receptor antagonist exhibited improved gut permeability ${ }^{[52-54]}$. According to all of these findings, Muccioli and coleagues hypothesized that the intestinal eCB system controls gut barrier function through a CB1-dependent mechanism ${ }^{[51]}$. However, plasma LPS levels have also been suggested as a factor related to the development of gut barrier dysfunction ${ }^{[55,56]}$. In an attempt to elucidate this problem, the cited authors applied simultaneously a CB1-receptor agonist (HU-210) and LPS, and observed a reduction in the mRNA expression of two tight junction proteins (occludin and ZO-1). In contrast, the CB2 receptor antagonist (SR144528) did not alter gut permeability ${ }^{[51]}$.

Gut permeability may play an important and precocious role in the pathophysiology of obesity and low-grade inflammation. In the last decades, the eCB emerged as a new metabolic pathway that could be involved in gut barrier dysfunc- tions, increasing gut permeability. As already mentioned, the eCB system comprehends bioactive lipids that bind to cannabinoid receptors eliciting cell signaling. The well-characterized lipids that elicit these responses are anandamide and 2-AG(57). Intrinsic eCBs mediators are synthesized by specific enzymes such as NAPE-PLD (N-acyl-phosphatidylethanolamine) and the effect of non-digestible nutrients with intrinsic prebiotic properties on host pathophysiology related to obesity seems to be important in this relationship. ECBs system acts by activation of protein-G-coupled receptors, namely $\mathrm{CB} 1$ and $\mathrm{CB} 2$ that are expressed in diverse patterns among different cell types(58). The effects of eCBs on metabolism are thought to be mediated primarily by $\mathrm{CB} 1$ and $\mathrm{CB} 2$ receptors. Studies have shown that stimulation of the eCB system increases food intake and that treatment with a $\mathrm{CB} 1$ antagonist in humans reduced food intake and decreases body weight, although detrimental effects on mood have been observed ${ }^{[59]}$.

In an experiment, Hind and colleagues demonstrated that anandamide (AEA) and oleoylethanolamide (OEA) (another eCB mediator) decreased blood brain barrier permeability and that this action was probably mediated by cannabinoid CB2 receptors, transient receptor potential vanilloid 1 (TRPV1) channels, calcitonin gene-regulated peptide (CGRP) receptor and PPAR $\alpha^{[60]}$. Moreover, it was recently proposed that the eCB system could be one of the ways that mediate communication between the gut and adipose tissue ${ }^{[51]}$.

The eCB system appears to link gut barrier function, adipose tissue composition and the gut microbiota, as previously demonstrated by Muccioly and co-workers. They showed that the eCB system was implicated in gut barrier alterations and increased gut permeability observed in obesity by observing that colonic CB1 mRNA levels were modified in animal models of gut microbiota modulation which included germ-free mice, antibiotic treatment, MyD88 (an important adaptor to Toll-like-4 receptor transduction) knockout mice, high-fat diet-treated mice and prebiotic-treated mice, suggesting that the gut microbiota selectively modulates $\mathrm{CB} 1$ colonic $\mathrm{mRNA}^{[51]}$.Therefore, there are strong evidences that the gut flora plays an important relationship with the permeability of the intestinal epithelium although the full mechanisms involved in this relationship still need further clarification. Among the hypotheses tested, the most accepted one is the role of LPS in the induction of chronic inflammation and in changing junctional proteins.

\section{Experimental studies related to intestinal permeability}

The importance of establishing the exact relationship between obesity and increased gut permeability is clear since damaging molecules such as LPS could be crossing the barrier through the paracellular route in obese subjects. In obese rats, the abnormal distribution of junctional proteins (such as zonulin and occludin) in the small intestine ${ }^{[29]}$, as well as the high inflammatory grade influenced by gut microbiota ${ }^{[30]}$, might support this hypothesis.

Animal studies have demonstrated morphological changes and increased gut permeability associated with obesity, but they did not indicate whether these changes occur in the small intestine or in the colon. In these models, it was suggested that there would be a change in the expression of junctional proteins of the intestinal epithelium, leading to greater permeation to endotoxins, as well as qualitative and quantitative changes in 
the crypts, villi and local enzymes ${ }^{[61,62]}$. However, in humans this relationship has yet to be demonstrated.

Other animal studies analyzed the morphology of the intestinal epithelium of diabetic rats and compared it to controls. Researchers showed that diabetic rats presented cell hypertrophy and hyperplasia at the small intestine, directly influencing the degree of nutrient absorption and, consequently, the pathophysiological process of diabetes mellitus ${ }^{[63-66]}$.

Besides the morphological alterations observed in most studies using diabetic rats, Adachi and co-workers ${ }^{[67]}$ found a significant increase in the expression and activity of the sucrase-isomaltase complex in the brush border of enterocytes in obese mice with hyperglycemia. Sucrase and isomaltase are both disaccharidases found in the small intestine epithelium and have a major role in the final stage of carbohydrate digestion.

In addition, Brun and co-workers showed that obese animals had a higher permeability of the intestinal barrier, a higher endotoxemia of the portal system and a higher level of proinflammatory cytokines in comparison to control animals. This finding reinforces the hypothesis of a possible link between obesity and intestinal morphofunctional changes ${ }^{[68]}$. Subsequently, these changes were observed in other animal studies that combined changes in gut microbiota composition and related them to an increased permeability and absorption of LPS ${ }^{[62,69]}$.

One of the ways we have to neutralize LPS action is through alkaline phosphatase activity. This is an important enzyme in intestinal homeostasis and, although its mechanism of action is not actually well understood, it is also known that it is able to detoxify LPS from gram negative bacteria and thus prevent translocation of these microorganisms into the bloodstream. De La Serre and colleagues tested obese diabetic rats and were able to observe a decrease in the activity of intestinal alkaline phosphatase in these animals, which could explain the increased levels of LPS ${ }^{[70]}$. Subsequently, Dyer and coworkers showed an increase in monosaccharide transporters in the intestines of diabetic subjects confirming that these alterations also occurred in humans ${ }^{[71]}$.

In summary, the understanding of the importance of the link between obesity and intestinal permeability and how it influences intestinal homeostasis may add knowledge to pathophysiology of obesity and metabolic diseases. Figure 2 summarizes this pathophysiology.

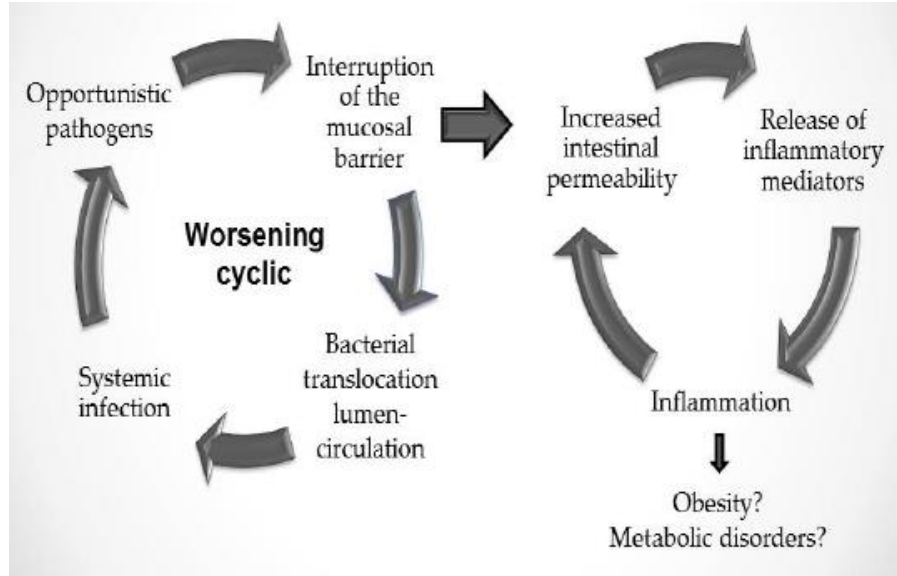

Figure 2: Intestinal homeostasis depends on intestinal permeability

\section{Clinical techniques to investigate gut permeability}

Most studies on gut permeability are performed in experimental models while in humans this evaluation is limited to techniques dependent on invasive procedures. Several studies have attempted to address this issue and currently the use of Ussing Chambers can bring new knowledge to this area. These devices are able to measure the electrical resistance through the mucosa of intestinal biopsies ${ }^{[72]}$. They provide a system to measure the transport of nutrients, ions and drugs across various epithelial tissues ${ }^{[73]}$. In this system, the two half chambers are separated by the epithelia (sheet of mucosa or monolayer of epithelial cells grown on permeable supports) and, additionally, electrodes are placed near the tissue. The ion movement through these barriers produces a difference in potential across the epithelium that is measured using voltage electrodes. Based on this premise, differences in potential reflect the movement of ions across the membrane and thus permeability.

The transport by renal epithelium influences what will be excreted in the urine and another way to indirectly investigate the absorptive capacity of the guts is through urinary dosage of previously ingested and absorbed substances. Ideally, a good marker of renal function may not suffer absorption or secretion by the renal tubules. Experimentally, this compound is represented by inulin, however, in clinical practice; the endogenous substance that is closer to the ideal is creatinine. In fact, the kidneys are the final determinants of what will be excreted since the glomerular filtrate can be quite modified when passing through the epithelium of renal tubules, where a wide variety of carriers are expressed. As a confounding factor in the study of renal excretion, it has yet been demonstrated that obesity alters expression of kidney transporters ${ }^{[74]}$. However, by studying renal excretion one can minimize the technical difficulty to investigate gut permeability in situ. The indirect measure of gut permeability by oral ingestion of some substances and the subsequent dosage of these compounds at the excreted urine involves the knowledge of the mechanism that allows the passages of molecules from the lumen across the epithelial membrane, called transepithelial transport. This transport is divided into transcellular transport (through the cells) and paracellular one (between the cells $)^{[75]}$. The size and solubility of the molecule and its regulation through junctional proteins are key points that define the substance's permeation route.

The basic rule in testing gut permeability by levels of urinary excretion is that, when there is an altered mucosal barrier which determines increased gut permeability, there will be a concomitant increase in the urinary excretion of the substance tested ${ }^{[76]}$. There are some substances that may be used for this and the polyethylene glycols, water soluble contrasts, radioactive labeled substances and sugars (mannitol, lactulose, sucrose, sucralose, xylose, cellobiose) are the most widely used ${ }^{[75]}$.

In humans, there are conflicting results in the literature regarding the use of these substances for evaluating gut permeability. Brignardello and coworkers analyzed obese subjects and compared them to normal weight controls and found no differences in the excretion of the above mentioned sugars ${ }^{[77]}$. In contrast, Teixeira and coworkers studied two groups of women, obese and controls, and demonstrated that obese women were able to excrete larger quantities of lactulose and mannitol than controls for the same amount of ingested sugars, suggesting thereby that obese women had increased gut permeability ${ }^{[78]}$. 
There are other indirect forms of evaluating gut permeability such as the measurement of myosin light chain phosphorilation in tissue samples of intestinal biopsies as well as the activity of myeloperoxidase which determines the degree of inflammation and neutrophilic permeation. Cytokines, such as tumour necrosis-alpha and interferon-gamma, are associated with an increased activity of the light chain myosin kinase, leading to the phosphorylation of myosin light chain and, as a result, cytoskeleton contraction. This contraction would cause a breakage of the "tight junctions" in the intestinal epithelium with resultant increased gut permeability. All of these changes were found in mice prone to obesity ${ }^{[70]}$ although clinically it has not yet been tested. Further studies are required in this field to determine a more accurate, easier, non-invasive method for assessing gut permeability.

The great difficulty to study the relationship between obesity and gut microbiota is that the majority of studies on this issue have cross-sectional design. Moreover, the majority of studies correlating changes in colonic intestinal microbiota and obesity have only analyzed the composition of these microorganisms, without testing its association to gut permeability. Indeed, less attention has been given to small intestine microbiota, possibly because of the difficulty in obtaining samples of this organ in humans ${ }^{[47]}$. However, it has been shown that the major gut permeability changes occur especially in the small intestine.

\section{Conclusions}

The association between obesity and increased gut permeability is still controversial despite current evidence suggesting that this association may be positive. Recent data showing that gut microbiota of obese and normal weight subjects differ from each other transformed the intestinal epithelium from something of unknown relevance and of little significance for the pathophysiology of obesity into a site that needs investigation. This investigation should focus both on the functional aspects of the intestinal epithelium itself and the composition of its microbiota. In this review, we tried to definitively establish the relationship between obesity, microbiota and gut permeability in humans. However, currently there is a lack of clinical data that prevents us to establish their exact association or to put the blame on one these factors. Therefore, there is much that we must find out so not only new techniques, but also new study designs, either cross-sectional or longitudinal and/or also interventional ones are needed to clarify the association between obesity, the microbiota and gut permeability.

Conflicts of Interest: The authors declare no conflict of interest.

\section{References}

1. Barrett, K.E. The world within--impact of the intestinal micobiota on whole body physiology and pathophysiology. (2009) J Physiol 587(Pt 17): 4151 .

2. Cani, P.D., Amar, J., Iglesias, M.A., et al. Metabolic endotoxemia initiates obesity and insulin resistance. (2007) Diabetes 56(7): 1761-172.

3. Ley, R.E., Turnbaugh, P.J., Klein, S., et al. Microbial ecology: human gut microbes associated with obesity. (2006) Nature 444(7122): 10221023.

4. Manco, M., Putignani, L., Bottazzo, G.F. Gut microbiota, lipopolysaccharides, and innate immunity in the pathogenesis of obesity and cardiovascular risk. (2010) Endocr Rev 31(6): 817-844.

5. Cani, P.D., Delzenne, N.M. The gut microbiome as therapeutic target. (2011) PharmacolTher 130(2): 202-212.

6. Reiquam, C.W., Allen, R.P., Akers, D.R. Normal and Abnormal Small Bowel Lengths: An Analysis of 389 Autopsy Cases in Infants and Children. (1965) Am J Dis Child 109: 447-451.

7. Treves, F. Lectures on the Anatomy of the Intestinal Canal and Peritoneum in Man. (1885) Br Med J 1(1264): 580-583.

8. Underhill, B.M. Intestinal length in man. (1955) Br Med J 2(4950): 1243-1246.

9. Backman, L., Hallberg, D. Small-intestinal length. An intraoperative study in obesity. (1974) Acta Acta Chir Scand 140(1): 57-63.

10. Guzman, I.J., Fitch, L.L., Varco, R.L., et al. (1977) Small bowel length in hyperlipidemia and massive obesity. Am J Clin Nutr 30(7): 1006-1008.

11. Ley, R.E., Backhed, F., Turnbaugh, P., et al. Obesity alters gut microbial ecology. (2005) Proc Natl Acad Sci U S A 102(31): 11070-11075.

12. Backhed, F., Ding, H., Wang, T., et al. The gut microbiota as an environmental factor that regulates fat storage. (2004) Proc Natl Acad Sci U S A 101(44): 15718-15723.

13. Turnbaugh, P.J., Ley, R.E., Mahowald, M.A., et al. An obesity-associated gut microbiome with increased capacity for energy harvest. (2006) Nature 444(7122): 1027-1031.

14. Lyra, A., Lahtinen, S., Tiihonen, K., et al. Intestinal microbiota and overweight. (2010) Beneficial microbes 1(4): 407-421.

15. Cammarota, G., Masucci, L., Ianiro, G., Bibbo, S., et al. Randomised clinical trial: faecal microbiota transplantation by colonoscopy vs. vancomycin for the treatment of recurrent Clostridium difficile infection. (2015) Aliment Pharmacol Ther 41(9): 835-843.

16. Vermeire, S., Joossens, M., Verbeke, K., et al. Donor species richness determines fecal microbiota transplantation success in inflammatory bowel disease. (2015) J Crohns Colitis.

17. Osada, T., Ishikawa, D., Watanabe, S. Fecal microbiota transplantation therapy for patients with gastrointestinal tract diseases. (2015) Nihon Shokakibyo Gakkai Zasshi 112(11): 1973-1981.

18. Cani, P.D., Delzenne, N.M. Gut microflora as a target for energy and metabolic homeostasis. (2007) Curr Opin Clin Nutr Metab Care 10(6): 729-734.

19. Nadal, I., Santacruz, A., Marcos, A., et al. Shifts in clostridia, bacteroides and immunoglobulin-coating fecal bacteria associated with weight loss in obese adolescents. (2009) Int J Obes (Lond) 33(7): 758767.

20. Santacruz, A., Marcos, A., Warnberg, J., et al. Interplay between weight loss and gut microbiota composition in overweight adolescents. (2009) Obesity (Silver Spring) 17(10): 1906-1915. 21. Duncan, S.H., Lobley, G.E., Holtrop, G., et al. Human colonic microbiota associated with diet, obesity and weight loss. (2008) Int J Obes (Lond) 32(11): 1720-1724.

22. Turnbaugh, P.J., Hamady, M., Yatsunenko, T., et al. A core guts microbiome in obese and lean twins. (2009) Nature 457(7228): 480-484.

23. Tsukumo, D.M., Carvalho, B.M., Carvalho-Filho, M.A., et al. Translational research into gut microbiota: new horizons in obesity treatment. (2009) Arq Bras Endocrinol Metabol 53(2): 139-144.

24. Cani, P.D., Amar, J., Iglesias, M.A., et al. Metabolic endotoxemia initiates obesity and insulin resistance. (2007) Diabetes 56(7): 1761-1772.

25. Zhang, C., Zhang, M., Wang, S., et al. Interactions between gut microbiota, host genetics and diet relevant to development of metabolic syndromes in mice. (2010) The ISME journal 4(2): 232-241.

26. Stappenbeck, T.S., Hooper, L.V., Gordon, J.I. Developmental regulation of intestinal angiogenesis by indigenous microbes 
via Paneth cells. (2002) Proc Natl Acad Sci U S A. 99(24): 15451-1545.

27. Backhed, F., Manchester, J.K., Semenkovich, C.F., et al. Mechanisms underlying the resistance to diet-induced obesity in germ-free mice. (2007) Proc Natl Acad Sci U S A 104(3): 979-984.

28. Fleissner, C.K., Huebel, N., Abd El-Bary, M.M., et al. Absence of intestinal microbiota does not protect mice from diet-induced obesity. (2010) Br J Nutr 104(6): 919-929.

29. Murphy, E.F., Cotter, P.D., Healy, S., et al. Composition and energy harvesting capacity of the gut microbiota: relationship to diet, obesity and time in mouse models. (2010) Gut 59(12): $1635-1642$.

30. Kovacs, P., Stumvoll, M. Fatty acids and insulin resistance in muscle and liver. (2005) Best Pract Res Clin Endocrinol Metab 19(4): 625-635.

31. Bugianesi, E., McCullough, A.J., Marchesini, G. Insulin resistance: a metabolic pathway to chronic liver disease. (2005) Hepatology 42(5): 987-1000.

32. Wei, Y., Chen, K., Whaley-Connell, A.T., et al. Skeletal muscle insulin resistance: role of inflammatory cytokines and reactive oxygen species. (2008) Am J Physiol Regul Integr Comp Physiol 294(3): 673-680.

33. Esteve, E., Ricart, W., Fernandez-Real, J.M. Gut microbiota interactions with obesity, insulin resistance and type 2 diabetes: did gut microbiote co-evolve with insulin resistance? (2011) Curr Opin Clin Nutr Metab Care 14(5): 483-490.

34. Piomelli, D. The molecular logic of endocannabinoid signalling. (2003) Nat Rev Neurosci 4(11): 873-884.

35. Engeli, S., Bohnke, J., Feldpausch, M., et al. Activation of the peripheral endocannabinoid system in human obesity. (2005) Diabetes 54(10): 2838-2843.

36. Liu, Y.L., Connoley, I.P., Wilson, C.A., et al. Effects of the cannabinoid CB1 receptor antagonist SR141716 on oxygen consumption and soleus muscle glucose uptake in $\mathrm{Lep}(\mathrm{ob}) / \mathrm{Lep}(\mathrm{ob})$ mice. Int (2005) J Obes (Lond) 29(2): 183-187.

37. Osei-Hyiaman, D., DePetrillo, M., Pacher, P., et al. Endocannabinoid activation at hepatic $\mathrm{CB} 1$ receptors stimulates fatty acid synthesis and contributes to diet-induced obesity. (2005) J Clin Invest 115(5): 1298-1305.

38. Pertwee, R.G. Cannabinoids and the gastrointestinal tract. (2001) Gut 48(6): 859-867.

39. Freund, T.F., Katona, I., Piomelli, D. Role of endogenous cannabinoids in synaptic signaling. (2003) Physiol Rev 83(3): 1017-1066.

40. Alger, B.E. Retrograde signaling in the regulation of synaptic transmission: focus on endocannabinoids. (2002) Prog Neurobiol 68(4): 247-286.

41. Wilson, R.I., Nicoll, R.A. Endocannabinoid signaling in the brain. (2002) Science 296(5568): 678-682.

42. Cota, D., Marsicano, G., Tschop, M., et al. The endogenous cannabinoid system affects energy balance via central orexigenic drive and peripheral lipogenesis. (2003) J Clin Invest 112(3): 423-431.

43. Browning, J.D., Horton, J.D. Molecular mediators of hepatic steatosis and liver injury. (2004) J Clin Invest 114(2): 147-152.

44. Pagotto, U., Marsicano, G., Cota, D., et al. The emerging role of the endocannabinoid system in endocrine regulation and energy balance. Endocr Rev 27(1): 73-100.

45. Bluher, M., Engeli, S., Kloting, N., et al. Dysregulation of the peripheral and adipose tissue endocannabinoid system in human abdominal obesity. (2006) Diabetes 55(11): 3053-3060.

46. Matias, I., Gonthier, M.P., Orlando, P., et al. Regulation, function, and dysregulation of endocannabinoids in models of adipose and beta-pancreatic cells and in obesity and hyperglycemia. (2006) J Clin Endocrinol Metab 91(8): 3171-3180.

47. Cote, M., Matias, I., Lemieux, I., et al. Circulating endocannabinoid levels, abdominal adiposity and related cardiometabolic risk factors in obese men. (2007) Int J Obes (Lond) 31(4): 692-699.

48. Hoareau, L., Buyse, M., Festy, F., et al. Anti-inflammatory effect of palmitoylethanolamide on human adipocytes. (2009) Obesity (Silver Spring) 17(3): 431-438.

49. Maccarrone, M., De Petrocellis, L., Bari, M., et al. Lipopolysaccharide downregulates fatty acid amide hydrolase expression and increases anandamide levels in human peripheral lymphocytes. (2001) Arch Biochem Biophys 393(2): 321-328.

50. Liu, J., Batkai, S., Pacher, P., et al. Lipopolysaccharide induces anandamide synthesis in macrophages via CD14/MAPK/ phosphoinositide 3-kinase/NF-kappaB independently of platelet-activating factor. (2003) J Biol Chem 278(45): 45034-45039. 51. Muccioli, G.G., Naslain, D., Backhed, F., et al. The endocannabinoid system links gut microbiota to adipogenesis. (2010) Mol Syst Biol 6: 392.

52. Cani, P.D., Possemiers, S., Van de Wiele, T., et al. Changes in gut microbiota control inflammation in obese mice through a mechanism involving GLP-2-driven improvement of gut permeability. (2009) Gut 58(8): 1091-1103.

53. Cani, P.D., Bibiloni, R., Knauf, C., et al. Changes in gut microbiota control metabolic endotoxemia-induced inflammation in high-fat diet-induced obesity and diabetes in mice. (2008) Diabetes 57(6): 1470-1481.

54. Cani, P.D., Neyrinck, A.M., Fava, F., et al. Selective increases of bifidobacteria in gut microflora improve high-fat-diet-induced diabetes in mice through a mechanism associated with endotoxaemia. (2007) Diabetologia 50(11): 2374-2383.

55. Kimura, H., Sawada, N., Tobioka, H., et al. Bacterial lipopolysaccharide reduced intestinal barrier function and altered localization of 7H6 antigen in IEC-6 rat intestinal crypt cells. (1997) J Cell Physiol 171(3): 284-290.

56. Sheth, P., Delos Santos, N., Seth, A., et al. Lipopolysaccharide disrupts tight junctions in cholangiocyte monolayers by a c-Src-, TLR4-, and LBP-dependent mechanism. (2007) Am J Physiol Gastrointest Liver Physiol 293(1): 308-318.

57. Lambert, D.M., Muccioli, G.G. Endocannabinoids and related $\mathrm{N}$-acylethanolamines in the control of appetite and energy metabolism: emergence of new molecular players. (2007) Curr Opin Clin Nutr Metab Care 10(6): 735-744.

58. Engeli, S. Central and peripheral cannabinoid receptors as therapeutic targets in the control of food intake and body weight. (2012) Handb Exp Pharmacol (209): 357-381.

59. Van Gaal, L.F., Rissanen, A.M., Scheen, A.J., et al. Effects of the cannabinoid-1 receptor blocker rimonabant on weight reduction and cardiovascular risk factors in overweight patients: 1-year experience from the RIO-Europe study. (2005) Lancet 365(9468): 1389-1397.

60. Hind, W.H., Tufarelli, C., Neophytou, M., et al. Endocannabinoids modulate human blood-brain barrier permeability in vitro. (2015) Br J Pharmacol 172(12): 3015-3027.

61. Brun, P., Castagliuolo, I., Di, L., et al. Increased intestinal 
permeability in obese mice: new evidence in the pathogenesis of nonalcoholic steatohepatitis. (2007) Am J Physiol Gastrointest Liver Physiol 292(2): 518-525.

62. Cani, P.D., Bibiloni, R., Knauf, C., et al. Changes in gut microbiota control metabolic endotoxemia-induced inflammation in high-fat diet-induced obesity and diabetes in mice. (2008) Diabetes 57(6): 1470-1481.

63. Stenling, R., Helander, H.F. Stereological studies on the small intestinal epithelium of the rat. 1. The absorptive cells of the normal duodenum and jejunum. (1981) Cell Tissue Res 217(1): 11-21.

64. Debnam, E.S., Ebrahim, H.Y., Swaine, D.J. Diabetes mellitus and sugar transport across the brush-border and basolateral membranes of rat jejunal enterocytes. (1990) J Physiol 424: 1325.

65. Zoubi, S.A., Mayhew, T.M., Sparrow, R.A. The small intestine in experimental diabetes: cellular adaptation in crypts and villi at different longitudinal sites. (1995) Virchows Arch 426(5): 501-507.

66. Fujita, Y., Kojima, H., Hidaka, H., et al. Increased intestinal glucose absorption and postprandial hyperglycaemia at the early step of glucose intolerance in Otsuka Long-Evans Tokushima Fatty rats. (1998) Diabetologia 41(12): 1459-1466.

67. Adachi, T., Mori, C., Sakurai, K., et al. Morphological changes and increased sucrase and isomaltase activity in small intestines of insulin-deficient and type 2 diabetic rats. (2003) EndocrJ 50(3): 271-279.

68. Brun, P., Castagliuolo, I., Di Leo, V., et al. Increased intestinal permeability in obese mice: new evidence in the pathogenesis of nonalcoholic steatohepatitis. (2007) Am J Physiol Gastrointest Liver Physiol 292(2): 518-525.

69. Cani, P.D., Delzenne, N.M. The role of the gut microbiota in energy metabolism and metabolic disease. (2009) Curr Pharm Des 15(13): 1546-1558.
70. de La Serre, C.B., Ellis, C.L., Lee, J., et al. Propensity to high-fat diet-induced obesity in rats is associated with changes in the gut microbiota and gut inflammation. (2010) Am J Physiol Gastrointest Liver Physiol 299(2): 440-448.

71. Dyer, J., Wood, I.S., Palejwala, A., et al. Expression of monosaccharide transporters in intestine of diabetic humans. (2002) Am J Physiol Gastrointest Liver Physiol 282(2): 241-248.

72. Osbak, P.S., Bindslev, N., Hansen, M.B. Relationships between body mass index and short-circuit current in human duodenal and colonic mucosal biopsies. (2011) Acta Physiol (Oxf) 201(1): 47-53.

73. Clarke, L.L. A guide to Ussing chamber studies of mouse intestine. (2009) Am J Physiol Gastrointest Liver Physiol 296(6): 1151-1166.

74. Briffa, J.F., Grinfeld, E., Jenkin, K.A., et al. Diet induced obesity in rats reduces NHE3 and $\mathrm{Na} / \mathrm{K}$-ATPase expression in the kidney. (2015) Clin Exp Pharmacol Physiol 42(10): 11181126.

75. Travis, S., Menzies, I. Intestinal permeability: functional assessment and significance. (1992) Clin Sci (Lond) 82(5): 471488.

76. DeMeo, M.T., Mutlu, E.A., Keshavarzian, A., et al. Intestinal permeation and gastrointestinal disease. (2002) J Clin Gastroenterol 34(4): 385-396.

77. Brignardello, J., Morales, P., Diaz, E., et al. Pilot study: alterations of intestinal microbiota in obese humans are not associated with colonic inflammation or disturbances of barrier function. (2010) Aliment Pharmacol Ther 32(11-12): 1307-1314.

78. Teixeira, T.F., Souza, N.C., Chiarello, P.G., et al. Intestinal permeability parameters in obese patients are correlated with metabolic syndrome risk factors. (2012) Clin Nutr 31(5): 735 740 .
Ommega Publishers

Journal Title: Journal of Gastrointestinal Disorder \& Liver Function (JGDLF)

Short name: J Gastro Dis Liver Func
ISSN No: 2471-0601

E-Mail Id: gastrology@ommegaonline.com

Website: www.ommegaonline.org 